PROCEEDINGS OF THE

AMERICAN MATHEMATICAL SOCIETY

Volume 128, Number 12, Pages 3609-3612

S 0002-9939(00)05504-0

Article electronically published on June 7, 2000

\title{
NOTE ON A LITTLEWOOD-PALEY INEQUALITY
}

\author{
J. MICHAEL WILSON
}

(Communicated by Albert Baernstein II)

\begin{abstract}
We show that a recent result of Littlewood-Paley type, due to the author, is essentially best-possible.
\end{abstract}

In a recent paper [W], the author proved Littlewood-Paley inequalities for certain finite linear sums $f=\sum_{I} \lambda_{I} \phi_{(I)}$, defined on $\mathbf{R}^{d}$. The summation here is indexed over $\mathcal{D}$, the family of dyadic cubes in $\mathbf{R}^{d}$; the $\lambda_{I}$ are complex numbers. The functions $\phi_{(I)}$, assumed to be smooth, belong to a family $\mathcal{F}$ that is "almost-orthogonal" and satisfies a mild decay condition. Precisely, we assume that there is an $M>d / 2$ such that for all $x \in \mathbf{R}^{d}$ and all $I \in \mathcal{D}$,

$$
\left|\phi_{(I)}(x)\right|+\ell(I)\left|\nabla \phi_{(I)}(x)\right| \leq|I|^{-1 / 2}\left(1+\left|x-x_{I}\right| / \ell(I)\right)^{-M} .
$$

The notation is more or less standard: $x_{I}$ denotes $I$ 's center, $\ell(I)$ is its sidelength, and $|I|$ is its Lebesgue measure (per tradition, we shall use $|E|$ to mean the Lebesgue measure of any measurable set $E$ ). We assume in addition that, for any finite linear $\operatorname{sum} \sum_{I} \gamma_{I} \phi_{(I)}$,

$$
\int_{\mathbf{R}^{d}}\left|\sum_{I} \gamma_{I} \phi_{(I)}\right|^{2} d x \leq \sum_{I}\left|\gamma_{I}\right|^{2}
$$

Recall that a non-negative $\sigma \in L_{l o c}^{1}\left(\mathbf{R}^{d}\right)$ is said to be an $A_{\infty}$ weight if there are positive constants $a$ and $b$ such that, for all cubes $Q \subset \mathbf{R}^{d}$ and measurable subsets $E \subset Q$,

$$
\frac{\int_{E} \sigma}{\int_{Q} \sigma} \leq a\left(\frac{|E|}{|Q|}\right)^{b}
$$

The main result from [W] is:

Theorem 1. Let $\mathcal{F}$ be a family satisying (1) and (2). Let $\rho>d$. For every $\sigma \in A_{\infty}$ as above and $0<p<\infty$, there is a constant $C=C(M, d, \rho, a, b, p)$ such that, for all finite linear sums $f=\sum_{I} \lambda_{I} \phi_{(I)}$ from $\mathcal{F}$,

$$
\int_{\mathbf{R}^{d}}|f|^{p} \sigma d x \leq C \int_{\mathbf{R}^{d}}\left(\sum_{I} \frac{\left|\lambda_{I}\right|^{2}}{|I|}\left(1+\left|x-x_{I}\right| / \ell(I)\right)^{-2 M+\rho}\right)^{p / 2} \sigma d x .
$$

Received by the editors August 24, 1998 and, in revised form, February 20, 1999.

2000 Mathematics Subject Classification. Primary 42B25.

Key words and phrases. Littlewood-Paley, weighted norm inequalities, Bochner-Riesz means.

(C)2000 American Mathematical Society 
The thing to watch is the exponent $-2 M+\rho$. If there were only one term in the sum, we could do no better than $-2 M$. The author finds it remarkable that, with such weak hypotheses on the family $\mathcal{F}$, one can get a result that seems so close to best-possible.

In this note we show that Theorem 1 is, in fact, within $\epsilon$ of best-possible.

Theorem 2. Theorem 1 fails for $p=2$ and all $\rho<d$.

Proof of Theorem 2. First we will give our original proof, which uses well-known facts about Bochner-Riesz kernels. The argument has the disadvantage of only working for $d>1$. At the end we will indicate how to extend the proof to $d=1$. The extension is easy, but it uses somewhat lesser-known facts about more general oscillatory kernels, due to Jurkat and Sampson [JS].

For $\delta>0$, let $K^{\delta}$ be the function whose Fourier transform is $\left(1-|\xi|^{2}\right)_{+}^{\delta}$. From St (pages 338 and 390), we have:

$$
\left|K^{\delta}(x)\right|+\left|\nabla K^{\delta}(x)\right| \leq C(1+|x|)^{-(\delta+(d+1) / 2)} .
$$

Asymptotic estimates for $K^{\delta}$ imply that, as long as $\delta<(d-1) / 2$, the convolution operator $K^{\delta} * f$ cannot be bounded on $L^{p}\left(\mathbf{R}^{d}\right)$ unless

$$
\frac{2 d}{d+1+2 \delta}<p<\frac{2 d}{d-1-2 \delta}
$$

in particular, for such $\delta$, convolution with $K^{\delta}$ must be unbounded on $L^{p}\left(\mathbf{R}^{d}\right)$ for large values of $p$.

We now prove a simple lemma.

Lemma. Let $\left\{I_{j}\right\}_{j}$ be the collection of dyadic cubes with sidelength equal to 1 . Suppose that, for each $j$, we have a function $h_{j}$ satisfying:

$$
\begin{gathered}
\operatorname{supp} h_{j} \subset I_{j}, \\
\int\left|h_{j}\right|^{2} d x \leq 1 .
\end{gathered}
$$

For each $j$, set $\phi_{j}=K^{\delta} * h_{j}$. Then, with $M=\delta+(d+1) / 2$, we have

$$
\left|\phi_{j}(x)\right|+\left|\nabla \phi_{j}(x)\right| \leq C\left(1+\left|x-x_{I_{j}}\right|\right)^{-M} ;
$$

and, for all finite linear sums $\sum_{j} \lambda_{j} \phi_{j}$,

$$
\int\left|\sum_{j} \lambda_{j} \phi_{j}\right|^{2} d x \leq \sum_{j}\left|\lambda_{j}\right|^{2}
$$

Proof of the Lemma. Inequality (3) follows from the bounds on $K^{\delta}$ given above. Inequality (4) follows because

$$
\int\left|\sum_{j} \lambda_{j} h_{j}\right|^{2} d x \leq \sum_{j}\left|\lambda_{j}\right|^{2}
$$

and $\left\|\widehat{K^{\delta}}\right\|_{\infty} \leq 1$

We will now suppose that Theorem 1 holds for some $\rho<d$, and see what happens. 
Let $f \in L^{2}\left(\mathbf{R}^{d}\right)$ have compact support. For each $j$, set $\gamma_{j}=\left(\int_{I_{j}}|f|^{2}\right)^{1 / 2}$. Define:

$$
h_{j}= \begin{cases}f \chi_{I_{j}} / \gamma_{j} & \text { if } \gamma_{j}>0, \\ 0 & \text { otherwise }\end{cases}
$$

and set $\phi_{j}=K^{\delta} * h_{j}$, as in the Lemma, where we choose $\delta>0$ to satisfy:

$$
\frac{\rho-1}{2}<\delta<\frac{d-1}{2}
$$

Inequality (5) implies that $2 M-\rho>d$ and that convolution with $K^{\delta}$ is unbounded on $L^{p}$ for large $p$. Let us fix a $p$ for which this is the case.

Note that $\left\{\phi_{j}\right\}_{j}$ is a finite collection, and that

$$
\sum_{j} \gamma_{j} \phi_{j}=K^{\delta} * f
$$

Let $s>1$ be the dual exponent to $p / 2$, and let $1<r<s$. Since we have already used $M$ to mean an exponent, we shall denote the Hardy-Littlewood maximal operator by $T$. Set $T_{r} g=\left(T\left(|g|^{r}\right)\right)^{1 / r}$. It is a standard fact that, if $g \not \equiv 0$, then $T_{r} g \in A_{\infty}$, with $A_{\infty}$ parameters only depending on $r$ and $d$. In fact, $T_{r} g$ belongs to the family $A_{1}$ : There is a constant $C=C(r, d)$ such that $T\left(T_{r} g\right) \leq C T_{r} g$ almost everywhere. One more fact we shall need: There is a constant $C=C(r, s, d)$ such that

$$
\left\|T_{r} g\right\|_{s} \leq C\|g\|_{s}
$$

for all $g \in L^{s}$.

If Theorem 1 held for the given $\rho$, then, for all $f$ as described and all $g \in L^{s}$, we would have:

$$
\begin{aligned}
\int\left|K^{\delta} * f\right|^{2} g d x & \leq \int\left|K^{\delta} * f\right|^{2} T_{r} g(x) d x \\
& \leq C \sum_{j}\left|\gamma_{j}\right|^{2} \int T_{r} g(x)\left(1+\left|x-x_{I_{j}}\right|\right)^{-2 M+\rho} d x \\
& \leq C \sum_{j}\left|\gamma_{j}\right|^{2} T\left(T_{r} g\right)\left(x_{I_{j}}\right) \\
& \leq C \sum_{j}\left|\gamma_{j}\right|^{2} \int_{I_{j}} T_{r} g d x \\
& \leq C \int\left(\sum_{j} \gamma_{j}{ }^{2} \chi_{I_{j}}\right) T_{r} g d x \\
& \leq C\left(\int\left(\sum_{j} \gamma_{j}{ }^{2} \chi_{I_{j}}\right)^{p / 2} d x\right)^{2 / p}\left\|T_{r} g\right\|_{s} \\
& \leq C\left(\int \sum_{j} \gamma_{j}{ }^{p} \chi_{I_{j}} d x\right)^{2 / p}\|g\|_{s} \\
& \leq C\|f\|_{p}^{2}\|g\|_{s},
\end{aligned}
$$

which would imply that the convolution with $K^{\delta}$ was bounded on $L^{p}\left(\mathbf{R}^{d}\right)$. This proves Theorem 2 when $d>1$. 
To handle $d=1$, we replace $K^{\delta}$ with a kernel $K_{\alpha, \beta}$, defined by:

$$
K_{\alpha, \beta}(t)=(1+|t|)^{-\beta} e^{i|t|^{\alpha}} .
$$

Here $\alpha$ and $\beta$ are positive numbers which will be chosen presently (depending on $\rho)$.

Notice that, if $\alpha$ and $\beta$ are both less than 1, then convolution with $K_{\alpha, \beta}$ must be unbounded on $L^{p}(\mathbf{R})$ when $p<\beta^{-1}$ (look at $K_{\alpha, \beta} * \chi_{B}$, where $B$ is a small interval centered at the origin); and, therefore, it must be unbounded on $L^{p}(\mathbf{R})$ for large $p$ 's. Notice also that, for such $\alpha$ and $\beta$, we have:

$$
\left|K_{\alpha, \beta}(t)\right|+\left|K_{\alpha, \beta}^{\prime}(t)\right| \leq C(\alpha, \beta)(1+|t|)^{-\beta}
$$

for all $t$. (Strictly speaking, $K_{\alpha, \beta}$ does not satisfy (6) at $t=0$, but this can be fixed by convolving $K_{\alpha, \beta}$ with a suitable mollifier: what is crucial here is the Lipschitz smoothness.)

We use the following result from [JS] (page 410):

Theorem 3. Let $\alpha, \beta$ be positive, $\alpha \neq 1, \beta<1$, and $(\alpha / 2)+\beta \geq 1$. Then convolution with $K_{\alpha, \beta}$ is bounded on $L^{p}(\mathbf{R})$ when

$$
\frac{\alpha}{\alpha+\beta-1} \leq p \leq \frac{\alpha}{1-\beta} \text {. }
$$

In particular, the convolution will be bounded on $L^{2}$.

It is easy to see that the one-dimensional analogue of the "simple lemma" goes through verbatim with $K_{\alpha, \beta}$ in place of $K^{\delta}$, and with $M \equiv \beta$. Now let $0<\rho<1$. Pick $0<\beta<1$, so close to 1 that $2 \beta-\rho>1$, and so there exists an $\alpha, 0<\alpha<1$, such that $(\alpha / 2)+\beta \geq 1$. Fix these $\alpha$ and $\beta$.

If Theorem 1 held for this $\rho$, then, by a virtual repetition of our earlier argument, convolution with $K_{\alpha, \beta}$ would be bounded on $L^{p}(\mathbf{R})$ for large $p$ 's.

Theorem 2 is proved.

\section{ACKNOWLEDGEMENT}

We must express our deep gratitude to the referee for several helpful comments; in particular, for directing us to the work of [JS (and others), which enabled us to extend our counterexample to the case $d=1$.

\section{REFERENCES}

[JS] W. B. Jurkat, G. Sampson, "The complete solution to the $\left(L^{p}, L^{q}\right)$ mapping problem for a class of oscillating kernels," Indiana University Mathematics Journal 30 (1981), 403-413. MR 84i:42033

[St] E. M. Stein, Harmonic Analysis, Princeton University Press, Princeton (1993).

[W] J. M. Wilson, "Global orthogonality implies local almost-orthogonality," to appear in Revista Matematica Iberoamericana.

Department of Mathematics, University of Vermont, Burlington, Vermont 05405 